\title{
RNA editing in plant mitochondria, cytoplasmic male sterility and plant breeding.
}

\author{
Alexandre Araya ${ }^{1}$ \\ Laboratoire REGER. EP 630. CNRS-Université Victor Segalen Bordeaux 2. 1 rue Camille Saint Saëns. 33077 Bordeaux cedex. France. \\ Eduardo Zabaleta \\ Present address: Allgemaine Botanik. Universitat Ulm. Albert Einstein Allee. D-89069 Ulm. Germany. \\ Valérie Blanc \\ Laboratoire REGER. EP 630. CNRS-Université Victor Segalen Bordeaux 2. 1 rue Camille Saint Saëns. 33077 Bordeaux cedex. France. \\ Present address: Howard Hughes Med. Inst. and Department of Molecular, Cell and Developmental Biology. University of California. Los Angeles. CA 90095-1662. USA. \\ Dominique Bégu \\ Laboratoire REGER. EP 630. CNRS-Université Victor Segalen Bordeaux 2. 1 rue Camille Saint Saëns. 33077 Bordeaux cedex. France. \\ Michel Hernould \\ Laboratoire de Biologie Cellulaire. Université Victor Segalen Bordeaux 2. Avenue des Facultés. 33405 Talence. France. \\ Armand Mouras \\ Laboratoire de Biologie Cellulaire. Université Victor Segalen Bordeaux 2. Avenue des Facultés. 33405 Talence. France. \\ Simon Litvak ${ }^{1}$ \\ Laboratoire REGER. EP 630. CNRS-Université Victor Segalen Bordeaux 2. 1 rue Camille Saint Saëns. 33077 Bordeaux cedex. France. \\ E-mail: simon.litvak@ibgc.u-bordeaux2.fr. \\ Work in our laboratory was supported by the CNRS, the University Victor Segalen Bordeaux 2, the Human Science Frontiers Program Organization (HSFPO), (RG-437/94 M), \\ the European Union (CI1*-CT93-0058), the Conseil Général d'Aquitaine, the French Ministère de l'Agriculture et de la Pêche, the France-Israel Program of Biotechnological \\ Applications to Agriculture.
}

RNA editing in plant mitochondria is a post-transcriptional process involving the partial change of $\mathrm{C}$ residues into $\mathrm{U}$. These $C$ to $U$ changes lead to the synthesis of proteins with an amino acid sequence different to that predicted from the gene. Proteins produced from edited mRNAs are more similar to those from organisms where this process is absent. This biochemical process involves cytidine deamination. The cytoplasmic male sterility (CMS) phenotype generated by the incompatibility between the nuclear and the mitochondrial genomes is an important agronomical trait which prevents inbreeding and favors hybrid production. The hypothesis that RNA editing leads to functional proteins has been proposed. This hypothesis was tested by constructing transgenic plants expressing a mitochondrial protein translated fom unedited mRNA. The transgenic "unedited" protein was addressed to the mitochondria leading to the appearance of mitochondrial dysfunction and generating the male sterile phenotype in transgenic tobacco plants. Male sterile plants were also obtained by expressing specifically a bacterial ribonuclease in the anthers. The economical benefits of artificially engineered male-sterile plants or carrying the (native) spontaneous CMS phenotype, implies the restoration to obtain fertile hybrids that will be used in agriculture. Restoration to fertility of transgenic plants was obtained either by crossing male-sterile plants carrying the "unedited" mRNA with plants carrying the same RNA, but in the antisense orientation or, in the case of plants expresing the ribonuclease, by crossing male-sterile plants with plants expressing an inhibitor specific of this enzyme.

\section{RNA editing}

Generalities. The first time the word "RNA editing" was used, a dozen years ago, concerned the insertion or deletion of uridine residues in the mitochondrial RNAs of some trypanosomes. After that, RNA editing has been found in mitochondria and chloroplasts from land plants, in the mitochondria of some fungi, in the nuclear-cytoplasmic compartments from animal cells and in the genomes of some viruses. The RNA editing process involves in some cases the modification of residues in RNA or, in other cases, the insertion and/or deletion of nucleotides in messenger RNA (for some reviews see Adler and Hadjuk, 1994; Araya et al., 1994; Schuster and Brennicke, 1994; Scott, 1995; Hanson et al., 1996; Smith et al., 1997; Stuart et al., 1997). The consequences of the RNA editing process are either the modification of the coded information for some amino acids or the generation of new initiation and/or termination codons. In organisms where RNA editing is active the protein sequence predicted from the gene may be different from that of the mRNA translated protein. While RNA editing is abondantly found in mitochondria and to a lesser extent in the chloroplast compartment of higher plants, it is not present in algae. RNA editing has also been descriibed in mitochondria from other organisms such as Physarum polycephalum, but not in yeast organelles. When detected in mammalian nuclear transcripts, it involves only punctual modifications. Finally, some animal viruses have also 


\section{INSERTION/DELETION}

$\mathrm{U}$ insertion/deletion

C insertion (rarely U, AA CU, GU, GC insertions) $G$ insertion

A insertion

3' mRNA poly A synthesis action

II. BASE MODIFICATION OR
REPLACEMENT
C to U
C to U

C to $\mathrm{U}$

C to $\mathrm{U}$

C to $\mathrm{U}$

U to $\mathbf{C}$

A to I

A to I

A to $\mathbf{G}$

$\mathbf{U}$ to $\mathbf{A}$

C to $A, A$ to $G \quad U$ to $G, U$ to $A$
Compartment/ Organism

Kinetoplastids (Trypanosoma, Leishmania, Crithidia)

Mitochondria. P. Polycephalum

Unknown mRNAs, rRNAs and tRNAstranscription

Paramyxoviruses

Ebola viruses

Mitochondria. Vertebrates

Land plants, Mitochondria,mRNAs, tRNAS, rRNAs

Chloroplasts, land plants, mRNAS

Physarum Polycephalum

Mammals, mRNAs (Apo-B)

Mammals and Marsupials

Land plants. (Mitochondria,

Chloroplasts), mRNA

Vertebrates, mRNAs(Glutamic and

serotonin receptor subunits)

Hepatitis Delta Virus

Drosophila, 4f-rnp

Humans, alpha-galactosidase

Unknown mRNA (Phe-to Tyr)

Acanthomoeba castellani, mitochondria, tRNAs
Mechanism and possible cofactors

Cleavage, ligation guide

RNAs....

Viral polymerase. Slippery

Viral polymerase. Slippery

transcription

Cleavage/TATase

\section{C-deamination}

Unknown

Unknown

Mooring sequence, Cytidine

deaminase (APOBEC-1)

Unknown

Transamination (?)

DRADA and other A-

deamination

A-deamination of the

antigenome

A to I deamination (?)

Base replacement. (?)

Table 1. Different types of RNA editing. More details which are not described in the text can be found in the reviews by (Araya et al., 1994; Schuster and Brennicke, 1994; Scott, 1995; Hanson et al., 1996; Smith et al., 1997; Stuart et al., 1997). This table has been adapted from Smith et al., 1997.

Two different types of RNA editing have been described. The first one involves the insertion or deletion of nucleotides with a change in the length of the final edited RNA product, and the second one where a base is changed to another without altering the size of the transcript.

The first case of RNA editing was discovered in 1986 and involved the addition or deletion of uridine residues in the RNA of kinetoplastid, the trypanosomal mitochondria (Benne et al., 1986). In certain cases up to $50 \%$ of the coding sequence of a given transcript may arise by this process. Important progress has been obtained in recent years on the elucidation of the mechanism of RNA editing in trypanosomes. The insertion or deletion of uridine regions is performed by a complex enzymatic system called the editosome and involves, in addition to a multimeric protein complex, a short nucleic acid called guide RNA (gRNA) which is partially complementary to the region neighbouring the site of editing (Blum and Simpson, 1992; Adler and Hadjuk, 1997) The gRNAs are encoded in the kinetoplast minicircles while true mitochondrial DNA is found only in few copies per organelle and carries the classical mitochondrial genes.
Insertion events of $\mathrm{G}$ and $\mathrm{C}$ residues in mRNAs of paramyxovirus (Vidal et al., 1990) and Physarum polycephalum mitochondria (Gott et al., 1993) have been described. The biochemical mechanism of base insertion in these organisms seems to be very different to those described in trypanosomes.

A second mechanism of RNA editing via base conversion has been described in higher mammals (Scott, 1995; Bass, 1997 and references therein), the virus of hepatitis D( Luo et al.,1990) and higher plants (Gualberto et al., 1989; Covello and Gray, 1989; Hiesel et al., 1989). Some examples of RNA editing are shown in Table I.

The case of the animal apolipoprotein B (apo-B) has been extensively studied. Apo-B is a protein found either as a very long polypeptide (Apo-B100) in the liver, or as only the amino terminal half (Apo-B48) in the intestine. The shortened protein APO-48 arises when a CAA triplet coding for glutamine is transformed to a UAA stop codon (Chen et al., 1987; Powell et al., 1987). An in vitro system able to use an unedited apo-B mRNA fragment as substrate allowed to determine the involvement of a cytidine deaminase activity in this reaction. More recently, it has been shown that the specificity of the cytidine deaminase activity, which is 
involved in apo-B mRNA editing, is due both to the presence of an RNA binding domain in this protein and to the anchor of the enzyme to a consensus sequence ("mooring sequence") found downstream of the unique editing site (Navaratnam et al., 1995).

Other RNA editing pathway reported in mammals concerns an A to I conversion in the subunits GluR-2, GluR-5 and GluR- 6 of the brain glutamate receptor (for reviews see Bass, 1995; Bass, 1997; and references therein). While the gene of these subunits has a triplet coding for glutamine (CAG), an arginine codon (CGG) is found at the same site of some transcripts. The in vitro analysis of the mRNA modification has shown that the $\mathrm{A}$ residue is changed to inosine by deamination. The I residue is «read» as a $\mathrm{G}$ during cDNA synthesis by reverse transcriptase. An ubiquitous enzyme, the adenosine deaminase (double stranded RNA adenosine deaminase or DRADA) which deaminates adenosine residues in double-stranded RNAs (dsRNA) plays an important role in the A to I change (Bass and Weintraub, 1988). DRADA was first known as an enzyme able to unwind dsRNAs by extensive deamination of A residues, thus destabilizing base paired regions of the dsRNA. The human adenosine deaminase cDNA has also been cloned. The cDNA sequence data revealed a strong degree of conservation across mammals (Kim et al., 1994). The C-end domain of human DRADA is similar to the catalytic region of other hydrolytic deaminases including the cytidine deaminase involved in apo-B mRNA editing (Navartnam et al., 1995). A putative zinc coordination site has been found in the same region, while in the middle domain of the protein, RNA binding motifs have been detected. Currently, it seems clear that DRADA is not the only adenosine deaminase found in vivo (Melcher et al., 1995). A cDNA encoding a new RED1 (RNA editase 1) able to specifically convert A to I at the Q/R editing site of GluR-2 was isolated, suggesting that a multifamily of dsRNA deaminases could be responsible for A to I changes in different tissues. The modification of the GluR-2 exon requires the involvement of the neighbouring intron to form the required double-stranded RNA substrate structure (Melcher et al., 1996). The important question whether other still unveiled RNA editing events take place in other tissues where this ubiquitous enzyme is present remains to be answered.

U-to-C conversion leading to the production of the viral protein p27 was suppossed to be the key step of RNA editing in hepatitis D virus (HDV) (Casey et al., 1992). However, a few years later it was found that the replication of this virus proceeds via an antigenome strand. In vitro studies using the antigenome strand as substrate have shown that animal cell DRADA is able to catalyze an A to I conversion at the same position where the $\mathrm{U}$ to $\mathrm{C}$ conversion was thought to occur in the viral genome (Casey and Gerin, 1995). RNA editing by the conversion of a UAG (stop) codon into a UIG (Trp) codon, plays an important role in the cycle of hepatitis D virus. This change allows the expression of a large and a small form of the HDV antigen from the antigenome. It is still unknown if the in vitro reaction can operate in vivo. An A to I editing has been observed in other animal substrates (references in Paul and Bass, 1998). The 3' end addition of A residues in vertebrate mitochondrial mRNAs are considered by some auhors as an RNA editing step since they may lead to the emergence of a termination codon.

\section{RNA editing in plant organelles}

This review is mostly focused on the RNA editing process observed in plant mitochondria. RNA editing in the organelles of higher plants involves a $\mathrm{C}$ to $\mathrm{U}$ transition. This process was first reported in angiosperms mitochondria in 1989 (Gualberto et al., 1989; Covello and Gray, 1989; Hiesel et al., 1989). The comparison between DNA and mRNA sequences of several mitochondrial genes showed some base changes in transcripts relative to the corresponding gene. In our laboratory we reached a similar conclusion by comparing the protein sequence of the subunit 9 (atp9) of the wheat mitochondrial ATP synthase (ATPase) and its corresponding gene (Graves et al, 1990; Bégu et al. 1990).

The crucial driving force that led to the discovery of RNA editing in plant mitochondria was the previous belief that the genetic code of plant organelles differed from the universal code. Analysis of the sequence of the cytochrome oxidase sub-unit II gene ( $\operatorname{cox}$ II) had shown that some extremely conserved tryptophan residues (UGG) of cox II were replaced by a CGG triplet which usually encodes the amino acid arginine (Fox and Leaver, 1981). However, it was intriguing that only a small fraction of the CGG triplets behave abnormally. In fact the $\mathrm{C}$ to $\mathrm{U}$ change in some $\mathrm{CGG}$ codons, through the RNA editing process, led to the expected UGG triplet universally coding for tryptophan showing that plant mitochondria use the universal genetic code. The complete sequence of the moss Marchantia polymorpha revealed that no editing process ocurred in this briophyte (Oda et al., 1992). Thus, the editing process was presumed to be confined to higher plants excluding mosses. However, recent results showed that RNA editing is also operating in chloroplasts and mitochondria of bryophytes and interestingly, an important number of $\mathrm{U}$ to $\mathrm{C}$ modifications were observed (Malek et al., 1996). As mentioned above when defining the RNA editing process, most of the editing sites reported corresponded to amino acids differing from those deduced from the gene as well as to the creation of initiation or stop codons. In addition, silent editing events not leading to a change of the encoded amino acid have been described in several plant mitochondrial mRNAs. The reason for the prevalence of silent editings is still obscure.

All mitochondrial RNAs may be edited although to very different degrees. While RNA editing is abondant in mitochondrial mRNAs, some $\mathrm{C}$ to $\mathrm{U}$ events involve organellar tRNAs and some intronic regions (MaréchalDrouard et al., 1993; Zanlungo et al., 1995). In the case of tRNA, editing is absolutely required to produce this macromolecule from the long precursor (Maréchal-Drouard et al.,1996; Marchfelder et al., 1996).

Incompletely edited transcripts have been detected. Soon after RNA editing discovery it was believed that only completely edited mRNA were translated into proteins as deduced from the analysis of the polysomal mRNA population (Gualberto et al., 1991) and the protein sequencing of the protein ATP9 (Bégu et al., 1991). However more recent results have shown that partially edited transcripts may be found in the polysomal fractions. Moreover, polymorphic proteins produced from partially edited mRNAs have been isolated in the case of maize mitochondrial rps12 (Lu et al. 1996). These authors have speculated that while the edited ribosomal protein is inserted 
in the ribosomal unit, the unedited form, found in the polysomal supernatant, may act as an RNA binding protein.

RNA editing implying a similar $\mathrm{C}$ to $\mathrm{U}$ conversion have also been found in some chloroplastic mRNAs. The observation that RNA editing was operating in chloroplasts came from the observation that the putative genes of some chloroplastic proteins lacked an initiation codon which could be created by a C to $\mathrm{U}$ change (Hoch et al., 1991). RNA editing in chloroplasts involves only a limited number of mRNAs.

\section{Mechanism of RNA editing in plant mitochondria}

RNA editing involving a C-to-U change may take place via different biochemical mechanisms:

(i) RNA cleavage followed by cytidine release, uridine incorporation and RNA ligation,

(ii) a cytidine deamination,

(iii) a transamination or

(iv) a transglycosilation.

It has been shown unambiguosly that RNA editing ocurred in many cases: apo-B, the brain glutamate receptor and the hepatitis $\mathrm{D}$ virus, via a deamination step catalyzed by a cytidine or an adenine deaminase.

To determine the biochemical mechanism of plant mitochondrial C-to-U RNA editing of the atp 9 transcript, an in vitro system was developed. The unedited substrate was obtained by in vitro transcription of the atp 9 gene using the T7 RNA polymerase system. A lysate obtained from highly purified wheat mitochondria was used as a source of RNA editing enzymes. Soluble proteins from the mitochondria lysate were able to catalyze the $\mathrm{C}$-to- $\mathrm{U}$ conversion of the unedited atp 9 substrate. More important was the observation that the in vitro edited mRNA products were modified at exactly the same positions found in vivo, thus confirming the fidelity of the cell-free reaction (Araya et al., 1992). Two approaches were use to detect the edited RNA product: i) the differential hybridization with an oligonucleotide probe complementary to the $3^{\prime}$ end of the atp 9 transcript carrying four edited sites; ii) the primer extension technique which had been used to study the in vitro apo-B mRNA editing mechanism.

Both approaches gave important information concerning the RNA editing process. Unfortunately, they were not suitable to study the enzymatic mechanism involved in this reaction. Thus, we settled a simplified in vitro system for routine assays which uses an in vitro radioactively labelled atp9 mRNA as substrate (Blanc et al., 1995; Blanc et al., 1996). After incubation with the mitochondrial lysate the edited product was extensively digested with nuclease P1 and submitted to a double dimension thin-layer chromatography (TLC) to separate the 5' NMPs. Using this approach we followed the RNA editing activity of the mitochondrial lysate after chromatographic steps on Mono Q, molecular sieve and heparin-agarose columns. The results obtained on the heparin column were interesting because no activity was found when each individual fraction of the eluate was tested. Nevertheless, a significant RNA editing activity was recovered after pooling all the chromatographic fractions, suggesting a multimeric nature of the RNA editing system. When the eluate of the heparin column was analyzed by gel retardation three major proteins and some minor ones were able to form a complex with the edited form of atp 9 mRNA, while atp9 DNA or tRNA were not retarded under the same conditions. Further studies allowed us to show that the major RNA binding proteins were apparently not involved in the RNA editing reaction, while a minor one was able to stimulate dramatically the RNA editing activity assayed in vitro as mentioned before (Blanc et al. 1996 and our unpublished results). These results strongly suggest that the plant mitochondrial RNA editing machinery contains more than one active protein and can be compared with the so called "editosome" complex involved in the trypanosomal and apolipoprotein-B RNA editing systems.

The search of an unambiguous answer concerning the biochemical mechanism of RNA editing in wheat mitochondria led us to perform the following experiment. When the atp 9 unedited transcript was double-labelled with $\left[\alpha-{ }^{32} \mathrm{P}\right] \mathrm{CTP}$ and $\left[5_{-}{ }^{3} \mathrm{H}\right] \mathrm{CTP}$ (the tritium label is in the base) and submitted to nuclease $\mathrm{P} 1$ digestion and bidimensional TLC, the ${ }^{32} \mathrm{P}$ and ${ }^{3} \mathrm{H}$ labels were found to comigrate with the UMP and CMP standards. Moreover, the ${ }^{3} \mathrm{H} /{ }^{32} \mathrm{P}$ ratio was the same in UMP and CMP. indicating that deamination of cytidine into uridine plays a key role in RNA editing in plant mitochondria (Blanc et al., 1995). These results are in good agreement with the in organello experiments described previously which showed that the $\alpha$-phosphate is retained during C-to-U conversion in plant mitochondrial RNAs (Rajasekhar et al., 1993). The latter approaches suggested that a deamination or a transamination mechanism may be involved in plant RNA editing. Although the double-labeling approach using partially purified mitochondrial extracts suggests strongly that a deamination process is operating in plant mitochondria, further experiments are necessary to ascertain that a transamination step is not involved in RNA editing. A similar double labeling in vitro approach using mitochondrial extracts obtained from etiolated pea seedlings by an independent group supported the role of cytidine deamination in plant mitochondrial RNA editing ( $\mathrm{Yu}$ and Schuster, 1995).

The analysis of multiple sequences surrounding editing sites in plant mitochondrial transcripts have not indicated the presence of a consensus sequence like the one described in the case of apo-B mRNA editing. We have obtained a significant degree of in vitro editing by using short fragments of atp and nad3 mRNAs containing respectively, 4 and 1 editing sites as substrates (Blanc et al., 1995). These results show that the specificity of the reaction is given by regions very near to the editing site. However, the question on the specificity allowing the editosome to choose only some of the multiple $\mathrm{C}$ residues in plant mitochondrial mRNAs to be transformed into $U$ remains elusive. Although there is no doubt that antisense RNAs fragments complementary to edited plant mitochondrial RNA regions are present in these organelles, no evidence exists that these antisense RNAs are involved in the RNA editing reaction. Our attemps to show the presence of an RNA guide-type of nucleic acid in the wheat mitochondrial lysate have failed. Thus, microccocal nuclease preincubation of the mitochondrial lysate and further inactivation of the nuclease did not affect the in vitro RNA editing efficiency (Araya et al., 1992). Moreover, extensive protease digestion of the wheat mitochondrial lysate and further end-labelling of a putative oligonucleotide present in this lysate failed to show any radioactive nucleic 
acid. Thus, our current hypothesis is that in the wheat mitochondrial RNA editing system the specificity to recognize the appropriate site may reside on the RNA binding proteins associated to the catalytic cytidine deaminase domain involved in the deamination reaction. However, the observation that RNA editing occurs in intronic regions and in the stem of plant mitochondrial tRNA by correcting a mispairing of the stem indicates that formation of double stranded RNA may be associated to the mechanism of RNA editing in these organelles (MaréchalDrouard et al., 1996; Marchfelder et al., 1996. Wissinger et al., 1991). Furthermore, extensive RNA editing has been related to the putative formation of double stranded structures in the $p s b L$ and $a t p B$ transcripts of hornworth chloroplasts (Bock et al., 1995; Yoshinaga et al., 1997).

As mentioned before very few $\mathrm{U}$ to $\mathrm{C}$ changes have been detected in plant mitochondrial edited transcripts. If the $\mathrm{U}$ to $\mathrm{C}$ changes have a functional significance two possibilities may be invoked to explain the biochemical mechanism of this reaction; either a reversible step of the reaction catalyzed by the cytidine deaminase, or a transamination step using an amino acid or other cofactor as the amino donor group.

\section{Sponteneous and artificially created cytoplasmic male sterility}

Cytoplasmic male sterility (CMS) is characterized by the suppression of the production of viable pollen and by the non-Mendelian inheritance of this trait. In contrast, the nuclear male sterility is herited following the Mendelian rules (for reviews see Levings, 1990; Hanson, 1991).

The best known example of this trait is the CMS observed in Zea mays $L$. carrying the Texas (T) cytoplasm (CMS-T). CMS was described for the first time in the early 1950's (Rogers and Edwardson; 1952). During the 1950s and 1960s, CMS-T was used extensively to avoid hand or mechanical emasculation in the production of hybrid maize thus allowing time shortening of the process and important cost reductions. However, corn leaf blight a severe pathology that struck maize carrying the T-cytoplasm (more than $85 \%$ of the USA surface was devoted to this plant) led to dramatical losses in the early 1970s. The blight was produced by a fungal pathogen Bipolaris maydis race $T$ which is more common in the southern regions of the USA with higher temperatures. The 1969 and 1970 epidemics showed the danger of the genetic vulnerability observed when a single, uniform plant cytoplasm is widely used. The molecular basis of the pathogen effect was shown to be related to the action of a newly synthesized chimeric protein (see below) in the membrane of the plant mitochondria since it led to respiration deficiency and a noticeable swelling (Levings and Siedow, 1992).

CMS has been observed in a wide variety of higher plants and is characterized by the very low level or the complete absence of pollen production. As CMS may have different causes linked to the mitochondria it may be assumed that it is the consequence of a mitochondrial dysfunction. The best studied case, at the biochemical and molecular level of a nucleo-cytoplasmic incompatibiliy leading to the apparition of the CMS phenotype, is the CMS-T of maize. In this case mitochondrial dysfunction and male-sterility are associated with mitochondrial DNA rearrangements creating a new ORF formed by fragments of rRNA genes. This ORF ( $T$ - urf13) is transcribed and translated into a chimeric polypeptide (Dewey et al., 1986). Other examples are the chimeric genes $p c f-S$ of petunia (Young and Hanson, 1987), orfB and orf224 of the "Polima" CMS in rapeseed (Handa et al., 1995). The transcripts of all these chimeric genes, with the exception of $T$-urf13, are edited only in the sequences formed by RNA fragments belonging to transcripts that are usually edited in vivo in the normal transcripts from the genes where they are expressed. Thus the chimeric proteins $p c f$ and $\operatorname{orf} B$ involved in petunia and rapesseed CMS are originated from edited transcripts since these fragments correspond to transcripts which are edited in vivo in the original whole length RNAs.

Mitochondrial genomes encoding chimeric proteins are presumably present in all tissues of the plant. It can be speculated that the CMS phenotype affects essentially the pollen producing organs because of the high requirement of energy by this tissue. Thus, a mitochondrial dysfunction produced by a chimeric protein interfering with the organelle function by different ways, will dramatically affect pollen production while other plant organs can overcome the consequences of mitochondrial dysfunction. A similar hypothesis has been proposed to explain the human mitochondrial pathologies found in high-energy consuming tissues like the muscle and the nervous tissue. An experiment was performed in transgenic plants both to test this hypothesis and to produce male sterile plants based on the mitochondrial-nucleus incompatibility.

The atp 9 gene is encoded in the plant mitochondrial genome. Assuming that the unedited form of atp 9 mRNA when translated into a protein should give a poorly or nonfunctional ATP synthase subunit, we constructed nuclear transgenic tobacco plants which have been transformed with a plasmid vector carrying either the edited or the unedited forms of atp 9 under the control of the constitutive cauliflower mosac virus (CaMV) promoter and terminator. The transgenic atp 9 gene was linked to a signal peptide allowing the targeting of the nuclear encoded and cytoplasmically synthesized ATP 9 protein subunit to the mitochondrial compartment. As seen in Figure 1, showing male sterile and fertile transgenic tobacco flowers, we demonstrated that a significant amount of plants showed the CMS phenotype when the unedited atp9 was used, while all transgenic plants carrying the edited form of atp 9 were fertile (Hernould et al., 1993).

Transgenic plants carrying the edited and unedited forms of atp 9 were shown to have integrated the gene at the nuclear level and to express atp9 at the transcriptional and translational level. The ATP9 protein was shown to be localized in the mitochondrial fraction of transgenic tobacco using an antibody against a fragment of the signal peptide which remains linked to ATP9 after the cleavage step necessary to enter into the mitochondria. The peptide tag was originated from a yeast gene which is absent in plant mitochondria and obviously in the native endogenous ATP9 subunit. No exogenous atp9 transcription or translation products were observed in transgenic plants that had been transformed with the unedited gene and which showed a fertile phenotype. Moreover, plants expressing the edited version of the transgene were all fertile. The latter results strongly support the idea that the transgenic ATP9 product is involved in the emergence of the CMS phenotype. 


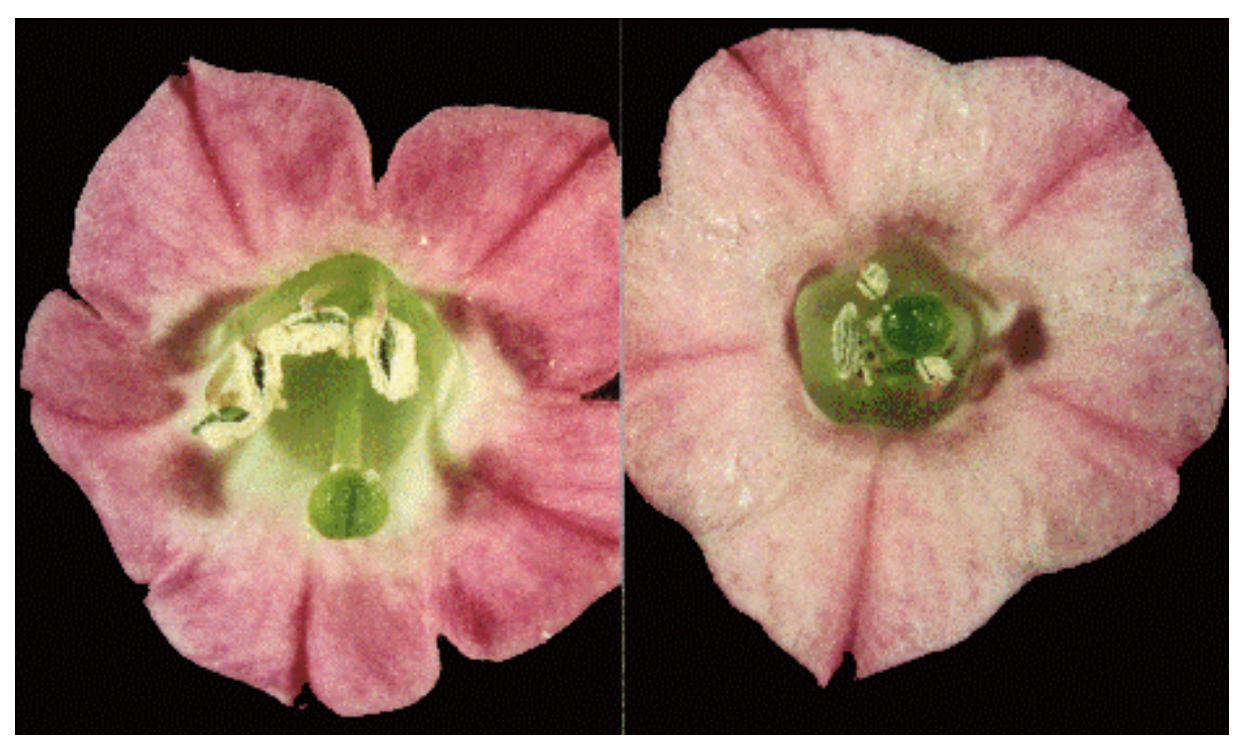

Figure . Wild-type and fertile transgenic tobacco plants. Male sterility was obtained by addressing an "unedited" mitochondrial protein to the organelle (Hernould et al., 1993)

It is interesting to mention that some genes can be found in the mitochondrial or the nuclear genome of highly related plant species. When the gene is found in the nucleus from one species, its sequence is more similar to the mitochondrial edited version than to the organellar gene sequence. We have speculated that the deleterious effect of the transgenic unedited ATP9 and the innocuousness of the edited form of ATP9 on mitochondrial function may explain the observation that genes are transferred from the mitochondrial to the nuclear compartment via an RNA intermediary, according to the endosymbiotyc hypothesis (Nugent and Palmer, 1991; Covello and Gray, 1992). Interestingly, electron microscopy showed that mitochondria in the tapetum were severely affected in transgenic tobacco carrying the nuclear unedited genes, while other tissues showed the conventional mitochondrial morphology. No mitochondrial modification was observed in transgenic plants carrying the edited mRNA. Respiration measurements of mitochondria isolated from transgenic plants expressing the unedited form of ATP9 gave significantly lower values than control mitochondria or those from plants expressing the edited ATP9 (Hernould et al., 1998).

To verify that the transgene is expressed in tapetal cells, experiments have been performed using the in situ hybridation and "priming in situ" (PRINS) techniques with oligonucleotides complementary to the CaMV terminator VI sequence as an in situ hybridization probe and as primer for the PRINS experiment. Both approaches confirmed that a significant level of unedited or edited atp 9 transcription is observed in the tapetum of transgenic plants.

It is important to emphasize that a general characteristic of CMS is the dysfunction of mitochondria in tapetal cells. It can be assumed that many situations leading to this situation may produce the CMS phenotype. Nevertheless, no clear relationship between the in vivo observed spontaneous CMS and a lower level of RNA editing can be established (Kurek et al., 1997). However, it has been reported that complete editing of an atp6 gene may restore the fertility of CMS rice (Iwabuchi et al., 1993). The production of chimeric protein, extensive recombination without creation of new ORFs, mitochondrial DNA deletions and eventually a decrease or lack of RNA editing may be some of the multiple causes of the CMS phenotype by lowering the capacity of the mitochondria to furnish energy to the cell.

\section{Recovering the fertility of artificially created male- sterile plants}

The approaches developed in more detail to produce transgenic CMS plants are: i) the expression of a protein such as the bacterial ribonuclease (Barnase). Barnase expressed in transgenic plants will specifically destroy the tissue where it is expressed. ii) the construction of nuclear transgenic plants carrying the information for the synthesis of "unedited" proteins addressed to the plant mitochondria as described in detail in the previous paragraph.

The use of Barnase to generate male-sterility is a drastic experimental approach that elegantly substitutes hand emasculation. Specific expression of this enzyme may be attained by using an anther specific promoter. After cell transformation, protein expression and plant regeneration, the active nuclease destroyed the pollen producing organs (Mariani et al., 1990). To restore fertility of male plants, the same authors crossed the sterile plants with transgenic plants expressing a specific inhibitor of Barnase. The formation of an inactive complex in the progeny between the Barnase and the specific inhibitor leaded to the restoration of fertility (Mariani et al., 1992).

Our approach to obtain male sterility was more subtle because it does not induce toxicity or a radical destruction of cells but intend to interfere with the physiological process of energy production. As detailed above male sterility was obtained by engineering plants able to synthesize an unedited mitochondrial protein in the cytosolic ribosomes which was 
further addressed to the mitochondria. Our approach to restore fertility was based on the antisense strategy (Zabaleta et al., 1996). The working hypthesis was that crossing the male-sterile plants produced as described above with plants expressing the same mRNA but in the antisense orientation should lead to the formation of a dsRNA. This dsRNA is suppossed to be substrate of an ubiquituous enzyme, the RNase III, which specifically cleaves dsRNAs. The digestion of this duplex carrying the mRNA which was at the origin of the male sterility trait should abolish this phenotype. As expected when we crossed a plant producing a high level of antisense atp 9 mRNA with a male-sterile counterpart expressing unedited RNA we obtained restoration to the fertile phenotype. In all cases, the restoration to male-fertility was associated with the complete loss of unedited atp9 mRNA. Very recently it was shown that the repression of the expression of the $55 \mathrm{kDa}, \mathrm{NADH}$-binding subunit of complex I was disturbed by antisense expression in transgenic potato plants. The effect of antisense was reflected by a dramatic decrease of pollen production and a lower male fertility (Heisert et al., 1997).

Work is under progress in our laboratory to produce the same type of CMS and fertility restored plants in other plants than tobacco by using an anther-specific promoter in order to increase the specificity of the whole process. Otherwise many questions are still unanswered concerning the existance of the RNA editing process. Although several evidences have been obtained indicating a strong relationship between RNA editing and the function of the protein issued from the edited mRNA, it is hard to understand why this high energy consuming process is still operating in the cell and why RNA editing is not found in some plants and in most of other organisms because the corresponding genome is already "edited".

\section{Acknowledgments}

The authors acknowledge the expert technical assistance of Ms Christina Calmels (supported by SIDACTION) and Mrs Evelyne Sargos. V. B. benefited from a pre-doctoral fellowship of the Ministère de la Recherche et de la Technologie, E. Z. benefited of a post-doctoral fellowship of the French Ministère des Affaires Etrangères. Several important references that should have been mentioned in this review have been ommited because of the length of the manuscript.

\section{References}

Adler, B. K., and Hadjuk, S. L. (1994) Mechanisms and origins of RNA editing. Current Opinion in Genetics and Development 4: 316-322.

Adler, B. K., and Hadjuk, S. L. (1997) Guide RNA requirement for editing-site specific endonucleolytic cleavage of preedited mRNA by mitochondrial ribonucleoprotein particles of Trypanosoma brucei. Molecular and Cellular Biology 17: 5377-5385.

Araya, A., Domec, C., Begu, D. and Litvak, S. (1992) An in vitro system for the editing of ATP synthase subunit 9 mRNA using wheat mitochondrial extracts. Proceedings of the National Academy of Sciences USA 89: 1040-1044.

Araya, A., Bégu, D., and Litvak, S. (1994) RNA editing in plants. Physiologia Plantarum 91: 543-550.
Bass, B. L., and Weintraub, H. (1988) An unwinding activity that covalently modifies its double-stranded RNA substrate. Cell 55: 1089-1098.

Bass, B. L. (1995) An I for editing. Current Biology 5: 598600 .

Bass, B. L. (1997) RNA editing and hypermutation by adenosine deamination. Trends in Biochemical Sciences 22: 157-162.

Bégu, D., Graves, P. V., Domec, C., Arselin, G., Litvak, S. and Araya, A. (1990) RNA editing of wheat mitochondrial ATP synthase subunit 9: direct protein and cDNA sequencing. The Plant Cell 2, 1283-1290.

Benne, R., Van Den Burg, J., Brakenhoff, J. P., Sloof, P., Van Boom, J.H., and Tromp, M. C. (1986). Major transcript of the frameshifted coxII gene from trypanosome mitochondria contains four nucleotides that are not encoded in the DNA. Cell 46: 819-826.

Blanc, V., Litvak, S., and Araya, A. (1995) RNA editing in wheat mitochondria proceeds by a deamination mechanism. FEBS Letters 373: 56-60.

Blanc, V. (1996) Mécanisme biochimique de l'édition des ARN dans les mitochondries de plantes. Thèse de Doctorat de l'Université Victor Segalen Bordeaux 2.

Blanc, V., Jordana, X., Litvak, S., and Araya, A. (1996) Control of gene expression by base deamination: the case of RNA editing in wheat mitochondria. Biochimie 78: 511-517.

Blum, B., and Simpson, L. (1992) Formation of guideRNA/mRNA chimeric molecules in vitro, the initial step of RNA editing is dependent on an anchor sequence. Proceedings of the National Academy of Sciences USA 89: 11944-11948.

Bock, R., and Maliga, P. (1995) In vivo testing of a tobacco plastid DNA segment for guide RNA function in psbL editing. Molecular and General Genetics 247, 439-443.

Casey, J. L., Bergmann, K. F., Brown, T. L., and Gerin, J. L. (1992) Structural requirements for RNA editing in hepatitis D virus: evidence for a uridine-to-cytidine editing mechanism. Proceedings of the National Academy Sciences USA 89: 7149-7153.

Casey, J. L., and Gerin, J. L. (1995) Hepatitis D virus RNA editing: Specific modification of adenosine in the antigenomic RNA. Journal of Virology 69: 7593-7600.

Chen, S. H., Habib, G., Yang, C. Y., Gu, Z. W., Lee, B. R., Weng, S. A., Silberman, S. R., Cai, S. J., Deslypere, J. P., Rosseneau, M., Gotto, A. M. Jr., Li, W. H., and Chan, L. (1987) Apoliprotein B-48 is the product of a mRNA with an organ-specific in-frame stop codon. Science 238: 363-366.

Covello P. S., and Gray M. W. (1989) RNA editing in plant mitochondria. Nature 341: 662-666.

Covello, P. S., and Gray, M. W. (1992) Silent mitochondrial and active nuclear genes for subunit 2 of cytochrome c 
oxidase (cox2) in soybean: evidence for RNA-mediated gene transfer. EMBO Journal 11: 3815-3820.

Dewey R. E., Levings, C. S. III, and Timothy, D. H. (1986) Novel recombinations in the maize mitochondrial genome produce a unique transcriptional unit in the Texas malesterile cytoplasm. Cell 44: 439-449.

Fox, T. D., and Leaver, C. J. (1981) The Zea mays mitochondrial gene coding cytochrome oxidase subunit II has an intervening sequence and does not contain TGA codons. Cell 26: 315-323.

Gott, J. M., Visomirski, L. M., and Hunter, J. L. (1993) Substitutional and insertional RNA editing of the cytochrome c oxidase subunit $1 \mathrm{mRNA}$ of Physarum polycephalum. The Journal of Biological Chemistry 268: 25483-25486.

Graves, P.V., Bégu, D., Velours, J., Neau, E., Belloc, F., Litvak, S., and Araya, A. (1990) Direct protein sequencing of wheat mitochondrial ATP-synthase subunit 9 confirms RNA editing in plants. Journal of Molecular Biology 214: 1-6.

Gualberto, J.M., Lamattina, L., Bonnard, G., Weil, J. H., and Grieneberger, J.M. (1989) RNA editing in wheat mitochondria results in the conservation of protein sequences Nature 341: 660-662.

Gualberto, J. M., Bonnard, G., Lammatina L., and Grienenberger, J. M. (1991) Expression of the wheat mitochondrial nad3-rps12 transcription unit: correlation between editing and $m R N A$ maturation. Plant Cell 3: 11091120 .

Handa H., Gualberto J. M., Grienenberger J. M. (1995) Characterization of the mitochondrial orfB gene and its derivative, orf 224 a chimeric open reading frame specific to one mitochondrial genome the Polima CMS in rapeseed (Brassica napus L ). Current Genetics 28: 546-552.

- Hanson, M. R. (1991) Plant mitochondrial mutations and male sterility. Annual Review of Genetics 25: 461-486.

Hanson, M. R., Sutton, C. A. , and Lu, B. (1996) Plant organelle gene expression: altered by RNA editing. (1996) Trends in Plant Sciences 1: 57-64.

Heisert V., Rasmusson A.G., Thick O., Brennicke A., and Grohman L. (1997) Antisense repression of the mitochondrial NADH-binding subunit of complex $\mathrm{I}$ in transgenic potato plants affects male fertility. Plant Science 127: 61-69.

Hernould, M., Suharsono, S., Litvak, S., Araya, A., and Mouras, A. (1993) Male sterility induction in transgenic tobacco plants with an unedited atp 9 mitochondrial gene from wheat. Proceedings of the National Academy of Sciences USA. 90: 2370-2374.

Hernould, M., Suharsono, Zabaleta, E., Carde, J. P., Litvak, S., Araya A., and Mouras, A. (1998) Impairment of tapetum and mitochondria in engineered male-sterile tobacco plants. Plant Molecular Biology 36: 499-508.
Hiesel, R., Wissinger, B., Schuster, W., and Brennicke, A. (1989) RNA editing in plant mitochondria. Science 246: 1632-1634.

Hoch, B., Maier, R. M., Appel, K., Igloi, G. L., and Kössel, H. (1991) Editing of a chloroplast mRNA by creation of an initiation codon. Nature 353: 178-180.

Iwabuchi, M., Kyozuka, J., and Shimamoto, K. (1993) Processing followed by complete editing of an altered mitochondrial atp6 RNA restores fertility of cytoplasmic male-sterile rice. EMBO Journal 12: 1437-1446.

Kim, U., Wang, Y., Sanford, T., Zeng, T., and Nishikura, K. (1994) Molecular cloning of cDNA for double-stranded RNA adenosine deaminase, a candidate enzyme for nuclear RNA editing. Proceedings of the National Academy of Sciences USA 91: 11457-11461.

Kurek, I., Ezra, D., Bégu, D., Erel, N., Litvak, S., and Breiman, A. (1997) Studies on the effect of nuclear background and tissue specificity on RNA editing of the mitochondrial ATP synthase subunits alpha, 6 and 9 in fertile and cytoplasmic male sterile (CMS) wheat. Theoretical and Applied Genetics 95: 1305-1311.

Levings , C. S. III. (1990) The Texas cytoplasm of maize: cytoplasmic male sterility and disease susceptibility. Science 250: 942-947.

Levings III, C. S., and Siedow, J. N. (1992) Molecular basis of disease susceptibility in the Texas cytoplasm of maize. Plant Molecular Biology 19: 135-147.

Lu, B., Wilson, R. K., Phreaner, C. G., Mulligan, R. M., and Hanson, M. R. (1996) Protein polymorphism generated by differential RNA editing of a plant mitochondrial rps 12 gene. Molecular and Cellular Biology 16: 1543-1549.

Luo, G., Chao, M., Hsieh, S.-Y., Sureau, C., Nishikura, K., and Taylor, J. (1990) A specific base transition occurs on replicating hepatitis delta virus RNA. Journal of Virology 64: 1021-1027.

Malek, O., Lättig, K., Hiesel, R., Brennicke, A., and Knoop, V. (1996) Mitochondrial RNA editing in bryophytes and molecular phylogeny of land plants. EMBO Journal 15: 1403-1411.

Marchfelder, A., Brennicke, A., and Binder, S. (1996) RNA editing is required for efficient excision of tRNA ${ }^{\text {Phe }}$ from precursors in plant mitochondria. The Journal of Biological Chemistry 271: 1898-1903.

Maréchal-Drouard, L., Ramamonjisoa, D. Cosset, A., Weil, J.-H., and Dietrich, A. (1993) Editing corrects mispairing in the acceptor stem of bean and potato mitochondrial phenylalanine tRNA. Nucleic Acids Research 21: 49094914 .

Maréchal-Drouard, L., Kumar, R., Remacle, C., and Small, I. (1996) RNA editing of larch mitochondrial tRNA ${ }^{\text {His }}$ precursors is a prerequisite for processing. Nucleic Acids Research 24: 3229-3234. 
Mariani, C., de Beuckeleer, M., Truttner, J., Leemans, J., and Goldberg, R. B. (1990) Induction of male sterility in plants by a chimaeric ribonuclease gene. Nature 347: 737-741.

Mariani, C., Gossele, V., de Beuckeleer, M., de Block, M., Goldberg, R. B., de Greef, W., and Leemans, J. (1992) A chimaeric ribonuclease inhibitor gene restores fertility to male-sterile plants. Nature 257: 384-387.

Melcher, T., Maas, S., Higuchi, M., Keller, W., and Seeburg, P. H. (1995) Editing of alpha-amino-3-hydroxy-5methylisoxazole-4-propionic acid receptor GluR-B premRNA in vitro reveals site-selective adenosine to inosine conversion. The Journal of Biological Chemistry 270: 85668570 .

Melcher, T., Maas, S., Herb, A., Sprengel, R., Seeburg, P. H., and Higuchi, M. (1996) A mammalian RNA editing enzyme. Nature 379: 460-464.

Navaratnam, N., Bhattacharya, S., Fujino, T., Patel, D., Jarmuz, A. L., and Scott, J. (1995) Evolutionary origins of apoB mRNA editing: catalysis by a cytidine deaminase that has acquired a novel RNA-binding motif at its active site. Cell 81: 187-195.

Nugent, J. M., and Palmer, J. D. (1991) RNA-mediated transfer of the gene cox II from the mitochondrion to the nucleus during flowering plant evolution. Cell 66: 473-481.

Oda, K., Yamato, K., Ohta, E., Nakamura, Y., Takmura, M., Nozato, N., Akashi, K., Kanegae, T., Ogura, Y., Kohshi, T., and Ohyama, K. (1992) Gene organization deduced from complete sequence of liverwort Marchantia polymorpha mitochondrial DNA: a primitive form of plant mitochondrial genome. Journal of Molecular Biology 223: 1-7.

Paul, M. S., and Bass, B. L. (1998) Inosine exists in mRNA at tissue specific levels and is most abondant in brain mRNA. EMBO Journal 17: 1120-1127.

Powell, L. M., Wallis, S. C., Pease, R. J., Edwards, Y. H., Knott, T. J., and Scott, J. (1987) A novel form of tissuespecific RNA processing produces apolipoprotein B-48 in the intestine. Cell 50: 831-840.

Rajasekhar, V. K., and Mulligan, R. M. (1993) RNA editing in plant mitochondria $\alpha$-phosphate is retained during $\mathrm{C}$-to- $\mathrm{U}$ conversion in mRNAs. The Plant Cell 5: 1843-1852.

Rogers, S. J., and Edwardson, J. R. (1952) The utilization of cytoplasmic male sterile inbreds in the production of corn hybrids. Agronomy Journal 44 : 8-13.

Schuster, W., and Brennicke, A. (1994) The Plant Mitochondrial Genome: Physical structure, information content, RNA editing and gene migration to the nucleus. Annual Review of Plant Physiology and Plant Molecular Biology 45: 61-78.

Smith, H. C., Gott, J. M., and Hanson, M. R. (1997) A guide to RNA editing, RNA 3: 1105-1123.

Stuart, K, Allen, T. E., Heideman, S., and Seiwert, S. D. (1997) RNA editing of kinetoplast protozoa. Microbiology and Molecular Biology Reviews. 61:105-120.
Scott, J. (1995) A place in the world for RNA editing. Cell 81: 833-836.

Vidal, S., Curran, J., and Kolakofsky D. (1990) A stuttering model for paramyxovirus $\mathrm{P}$ mRNA editing. EMBO Journal 9: 2017-2022.

Wissinger, B., Schuster, W., and Brennicke, A. (1991) Transplicing in Oenothera mitochondria: nad 1 mRNAs are edited in exon and trans splicing group II intron sequences. Cell 65: 473-482.

Yoshinaga, K., Kakehi, T., Shima, Y., Iinuma, H., Masuzawa, T., and Ueno, M. (1997) Extensive RNA editing and possible double-stranded structures determining editing sites in the atpB transcripts of hornwort chloroplasts. Nucleic Acids Research 25: 4830-4834.

Young, E. H., and Hanson, M. R. (1987) A fused mitochondrial gene associated with CMS is developmentally regulated. Cell 50: 41-49.

$\mathrm{Yu}$, W., and Schuster, W. (1995) Evidence for the site specific deamination involved in C-to-U RNA editing of plant mitochondria. The Journal of Biological Chemistry 270: $18227-18233$.

Zabaleta, E., Mouras, A., Hernould, M., Suharsono and Araya, A. (1996). Transgenic male-sterile plant induced by an unedited atp 9 gene is restored to fertility by inhibiting its expression with antisense RNA. Proceedings of the National Academy of Sciences USA 93: 11259-11263.

Zanlungo, S., Quiñones, V., Moenne, A., Holuigue, L. and Jordana, X. (1995) Splicing and editing of rps10 transcripts in potato mitochondria. Current Genetics 31: 647-656. 